\title{
Investigational Product Site Release Document
}

National Cancer Institute

\section{Source}

National Cancer Institute. Investigational Product Site Release Document. NCI

Thesaurus. Code C115710.

Records describing the approval for a site to receive a supply of investigational medicinal products, or drugs. 